\title{
Integrated Scheduling for Steelmaking Continuous Casting- Hot Rolling Processes considering Hot Chain Logistics
}

\author{
Sen Wang $\mathbb{D}^{1,2}$ Yarong Shi, ${ }^{3}$ and Shixin Liu $\mathbb{D}^{3}$ \\ ${ }^{1}$ College of Electronic and Information Engineering, Tongji University, Shanghai 201804, China \\ ${ }^{2}$ Shanghai Baosight Software Limited Company, Shanghai 201900, China \\ ${ }^{3}$ College of Information Science \& Engineering, Northeastern University, Shenyang 110819, China
}

Correspondence should be addressed to Sen Wang; wangsen@baosight.com

Received 11 November 2019; Revised 8 February 2020; Accepted 13 February 2020; Published 4 May 2020

Academic Editor: Leandro F. F. Miguel

Copyright (C) 2020 Sen Wang et al. This is an open access article distributed under the Creative Commons Attribution License, which permits unrestricted use, distribution, and reproduction in any medium, provided the original work is properly cited.

Steelmaking continuous casting (SCC) - hot rolling (HR) is a key process in the production of steel products. It is also a process with large energy consumption. Energy saving has always been an important goal of production scheduling of this process. In this paper, aiming at integrated scheduling optimization for SCC-HR processes, energy saving objective is converted to minimize waiting time of slabs in slab yard, so as to reduce slab temperature loss and achieve the goal of saving energy. An integrated twostage mathematical programming model is established for the above problems, and a hybrid algorithm of genetic algorithm and linear programming is designed for the integrated model. The correctness of the model and the validity of the algorithm are verified by computational experiments using simulated instances.

\section{Introduction}

Steel production processes are high energy consumption and pollution processes. Energy conservation and emission reduction have always been an important goal that both practitioner and academia concern and research. Steelmaking continuous casting (SCC) - hot rolling (HR) is a key process in steel production, which belong to two operating areas: SCC area and HR area. These two areas are connected by a slab yard, as shown in Figure 1. SCC processes are organized by mass production, including three processes of steel making, refining, and continuous casting. Production organization of steel making and refining process is in unit of heat. The weight of molten steel per heat is about $100 \mathrm{t}-300$ tons, and the temperature of molten steel is about $1600^{\circ} \mathrm{C}$. Production organization in the continuous casting process is in unit of cast, and each cast is composed of several heats to tens heats with the same steel grade. During steelmaking and refining processes, firstly, melted steel is discharged from a basic oxygen furnace (BOF) into a steel ladle. Then, the steel ladle is transported to refining station, such as ladle furnace (LF) and/or RH furnace, to adjust steel composition to specified steel grade. Finally, the steel ladle is transported to a continuous caster (CC) for multiheat continuous casting, where the melted steel is cast into slabs and delivered to slab yard. In HR area, the selected slabs are loaded into reheating furnace in the order of rolling sequence, where slabs are reheated to $1150^{\circ} \mathrm{C}$ to $1300^{\circ} \mathrm{C}$ and then pushed into hot rolling mill to produce hot rolling strips of different mechanical properties.

Energy consumed by slab reheating accounts for $80 \%$ of the total energy consumption of HR processes, while energy consumption of the rolling mill is only $16.9 \%$ [1]. Reheating of a 25 ton slab to $1250^{\circ} \mathrm{C}$ from atmospheric temperature consumes approximately $1000 \mathrm{~m}^{3}$ of gas [2]. Therefore, there is a large amount of energy loss due to cooling during slabs stay in yard. Making use of integrated scheduling to improve logistics connection, optimize production rhythm, and reduce energy consumption caused by slab reheating is an effective way to reduce energy consumption during SCC-HR processes.

In theory, integrated scheduling problem of SCC-HR processes is one kind of complex combinatorial optimization problem with important academic research value. Therefore, it has always attracted attention and research [3-5]. 


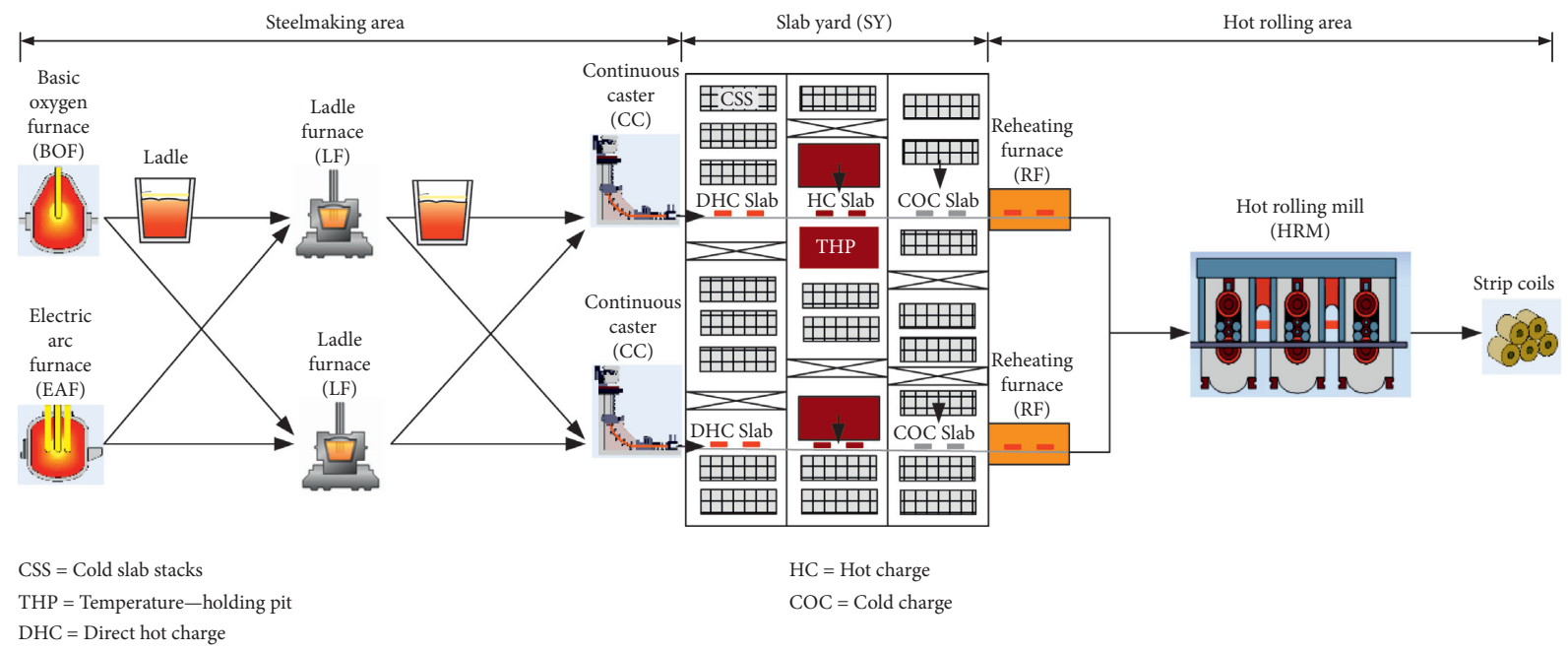

FIGURE 1: Basic steelmaking continuous casting (SCC) - hot rolling (HR) production processes.

Research on scheduling of SCC-HR processes mainly includes flexible flow shop scheduling for SCC process, hot rolling scheduling for HR process, and integrated scheduling for SCC-HR processes.

For problems of flexible flow shop scheduling in SCC process, optimization approaches include mathematical programming [6-9], metaheuristics which includes artificial bee colony algorithm [10], evolutionary programming [11], tabu search [12], particle swarm optimization algorithm [13], bilayer optimization approaches [14], two-phase heuristic algorithm [15], and hybrid algorithm [16]. Considering the uncertainty of the SCC process, Ye et al. [17] proposed a robust optimization method, and Hao et al. [18] proposed a soft-decision approach based on two-layered scheduling.

With increasing importance of environmental issues, scholars have been paying more attention to optimization goal of reducing energy consumption. Considering ladder price environment, Tan et al. [19] formulated a mathematical programming model to minimize electricity cost and proposed a two-stage algorithm to solve the model. Tan and Liu [20] established a hybrid model of mathematical programming and constrained programming for the same kind of problem and proposed a heuristic algorithm combined with constraint propagation optimization method. Hadera et al. [21] developed a mixed-integer linear programming model and proposed a bi-level heuristic algorithm to tackle instances of industrial size for the optimization of electricity consumption in a stainless steel plant. Gajic et al. [22] presented an integrated scheduling and electricity optimization system that can help melt shop to adjust its production schedule to volatile electricity prices and thus lower its costs.

HR process takes slabs as a basic production unit. Hot rolling scheduling is to select slabs and determine their rolled sequence under constraints of rolling process. The main methods for hot rolling scheduling are to formulate the problems as multiple traveling salesman problems, vehicle routing problems, or prize collecting vehicle routing problems [23] and then design optimization algorithms to solve the models. Optimization algorithms include mathematical programming $[24,25]$, metaheuristics which include particle swarm optimization [26], tabu search [27], scatter search [28], hybrid evolutionary algorithms [29], two-stage scheduling method [30,31], multiobjective ant colony optimization algorithm [32], and guided local search algorithm [33].

With increasing importance of energy saving and emission reduction, the integrated scheduling problem for SCC-HR processes has become focus of this research field. The main goal of integrated scheduling is to reduce energy consumption of the whole processes by coordinating production rhythm of SCC process and HR process, so as to reduce time interval from the moment of slab cutting to start time of reheating process and hence reduce temperature loss of slab during waiting in slab yard. Xu et al. [34] established a generic model for integrated scheduling problems of SCCHR processes and proposed three coordination heuristics. These heuristics are discussed and compared for simplified case studies. Computational results show the advantage of their new intersection coordination heuristic among these three coordination approaches in terms of solution quality and computational effort. Tan et al. [35] established an integrated scheduling model with the objective of maximizing the number of slabs processed in the hot charge rolling or direct hot charge rolling mode. Using the decomposition strategy, they proposed a hybrid algorithm of mathematical programming and constraint programming. Lin et al. [36] introduced a concept named 'order-set' and constructed a multiobjective optimization model with interval-valued objective functions for the integrated production planning problems in SCC-HR processes. To solve the multiobjective problems, they proposed an approach based on a modified interval multiobjective optimization evolutionary algorithm. Using the concept "order-set," Lin et al. [37] established an integrated multiobjective order planning model for SCC-HR processes with consideration of four conflicting objectives. To tackle the multiobjective model, the author proposed a novel knee point-driven many-objective global-best harmony search algorithm. 
Mattik et al. [38] established a mixed-integer linear optimization model based on the block planning principle for each process of SCC and HR and presented a hierarchical scheduling approach to develop production schedules that take technological sequences of SCC and HR programmes into account.

The integrated scheduling problem is hard to solve due to the following difficulties: (1) there is a natural contradiction between mass production of SCC-HR processes and multivarieties and small batch demand of customer orders. In SCC process, due to the constraints of production process, molten steel of the same steel grade is usually organized into large-batch production of hundreds of tons even thousands of tons. However, customer orders range from tons to tens of tons. Moreover, there are various requirements for mechanical properties of products, which in turn require different steel grades to meet their needs. This contradiction leads to great difficulties to the integrated scheduling problem. (2) The basic production units of SCC process and HR process are different. In SCC process, heat is the basic production unit, and the molten steel is about 100-300 tons per heat. In HR process, slab is the basic production unit, and the weight of a slab is about 20-30 tons. This inconsistency of production units makes modelling and optimizing very difficult. (3) Production rhythm of SCC process and HR process are not consistent. It takes about 4 mins to produce a slab in SCC process. It takes 2 mins to roll a slab to strip. (4) The integrated model generally belongs to large-scale integer programming model, which is hard to solve optimally.

In this paper, aiming at the integrated scheduling optimization for SCC-HR processes, energy saving objective is converted to minimize waiting time of slabs in slab yard. The optimization goal is to minimize total waiting time of slabs in slab yard, so as to reduce temperature loss of slabs and saving energy consumption. A two-stage mathematical programming model is established and then is integrated. A hybrid algorithm based on genetic algorithm and linear programming is designed to solve the integrated model. The correctness of the model and the effectiveness of the algorithm are verified by computational experiments.

The following parts of the paper are organized as follows. In Section 2, a mixed integer programming model and a linear programming model are established for SCC process and HR process, respectively. Then, the two models are integrated into one. Section 3 proposes two algorithms to solve the integrated model. One is a two-stage heuristic algorithm, and the other is a genetic algorithm based on the aforementioned two-stage heuristic algorithm. Section 4 tests the model and algorithms using simulated instances. The computational results show that the models and algorithms solve the problem effectively. Section 5 gives conclusions and discusses further research directions.

\section{Problem Description and Mathematical Models}

The integrated scheduling problem for SCC-HR processes can be divided into a flexible flow shop scheduling problem in SCC process and a single machine scheduling problem in HR process. The two types of scheduling problems are coupled with each other and need to be integrated optimization. According to the actual investigation on SCC-HR processes of a large iron and steel plant in China, the following assumptions are made in this study:

(1) In SCC process, continuous casters that execute each cast have been assigned. The problem to be solved in the flexible flow shop scheduling is assigning steel making and refining devices for each heat, and determining the start time of each heat in every processing stages.

(2) In HR process, the slabs rolled sequence in HR process has been determined, and it is necessary to determine the charging time of each slab in hot rolling plan.

2.1. Nomenclature. The indices, sets, parameters, and decision variables used in this paper are listed in Table 1.

\subsection{Mathematical Models}

2.2.1. Mathematical Model for SCC Process. The flexible flow shop scheduling problem in SCC process is NP-hard. The main decisions involved in this problem are assigning and sequencing heats on machines and determining start time for all the heats. The binary variable $x_{i m}$ is an assignment variable, and binary variable $y_{i i^{\prime}}$ is a sequencing variable. The continuous variable $t_{i s}$ denotes start time of heat $i$. Referring to the model of Jain and Grossmann [39], the following mixed integer linear problem (MILP) model is established for the flexible flow shop scheduling problem:

$$
\begin{aligned}
& \min \quad C_{1}=\max _{i \in I, m \in M^{4}}\left\{t_{i 4}+\sum_{m \in M^{4}} x_{i m} p_{i m}\right\}, \\
& \text { s.t. } \quad \sum_{m \in M^{s}} x_{i m}=1, \quad \forall i \in I, s=1,2,3,4, \\
& y_{i i^{\prime}}+y_{i^{\prime} i} \geq x_{i m}+x_{i^{\prime} m}-1, \quad \forall i, i^{\prime} \in I, i^{\prime}>i, m \in M^{s}, s=1,2,3,4, \\
& y_{i i^{\prime}}+y_{i^{\prime} i} \leq 1, \quad \forall i, i^{\prime} \in I, i^{\prime}>i,
\end{aligned}
$$




$$
\begin{gathered}
y_{i i^{\prime}}+y_{i^{\prime} i}+x_{i m}+x_{i^{\prime} m^{\prime}} \\
\leq 2, \quad \forall i, i^{\prime} \in I, i^{\prime}>i, m, m^{\prime} \in M^{s}, m \neq m^{\prime}, s=1,2,3,4, \\
t_{i(s+1)}-t_{i s}-p_{i m} x_{i m} \geq T_{m, m^{\prime}}^{\min }-\operatorname{big} M\left(2-x_{i m}-x_{i m^{\prime}}\right), \quad \forall i \in I, m \in M^{s}, m^{\prime} \in M^{s+1}, s=1,2,3, \\
t_{i(s+1)}-t_{i s}-p_{i m} x_{i m} \leq T_{m, m^{\prime}}^{\max }+\operatorname{big} M\left(2-x_{i m}-x_{i m^{\prime}}\right), \quad \forall i \in I, m \in M^{s}, m^{\prime} \in M^{s+1}, s=1,2,3, \\
t_{i^{\prime} s} \geq t_{i s}+p_{i m} x_{i m}-\operatorname{big} M\left(1-y_{i i^{\prime}}\right), \quad \forall i, i^{\prime} \in I, m \in M^{s}, s=1,2,3,4, \\
t_{i^{\prime} 4} \geq t_{i 4}+p_{i m} x_{i m}-\operatorname{big} M\left(1-a_{i i^{\prime}}\right), \quad \forall i, i^{\prime} \in I, m \in M^{4}, \\
t_{i^{\prime} 4} \leq t_{i 4}+p_{i m} x_{i m}+\operatorname{big} M\left(1-a_{i i^{\prime}}\right), \quad \forall i, i^{\prime} \in I, m \in M^{4}, \\
t_{i s} \geq 0, \quad \forall i \in I, m \in M^{s}, s=1,2,3,4, \\
x_{i m}, y_{i i^{\prime}} \in\{0,1\}, \quad \forall i, i^{\prime} \in I, m \in M^{s}, s=1,2,3,4,
\end{gathered}
$$

where objective (1) minimizes the makespan of SCC process. Constraint (2) ensures that each heat needs exactly one machine for processing in each stage. Constraint (3) is a logical relationship between assignment and sequencing variables. The underlying logic behind this constraint is that if heat $i$ and $i^{\prime}$ are assigned to machine $m$, then they must be processed one after the other. Constraints (4) and (5) are logical cuts. The former is based on the logic that either $i$ is processed before $i^{\prime}$ or vice versa. The latter ensures that sequencing variables are zero if $i$ and $i^{\prime}$ are assigned to different machines. Constraints (6) and (7) ensure that transfer time between continuous processes of a heat is in time interval. Constraint (8)-(10) is the sequencing constraint. It ensures that if the sequencing variable $y_{i i^{\prime}}$ (or parameters $\left.a_{i i^{\prime}}\right)$ is one, then heat $i^{\prime}$ is processed after heat $i$.

2.2.2. Mathematical Model for HR Process. The scheduling problem of HR process is relatively simple. Since the rolling sequence is known, only the charging start time of each slab needs to be determined. A linear programming model is established for this problem, which is as follows:

$$
\begin{aligned}
& \min \quad C_{2}=\max _{j \in J}\left\{s_{j}+h_{j}+p_{j}\right\}, \\
& \text { s.t. } \quad s_{j}-c_{j} \geq T S, \quad \forall j \in J, \\
& \left(s_{j^{\prime}}+h_{j^{\prime}}\right)-\left(s_{j}+h_{j}\right) \geq p_{j}+Q-\operatorname{big} M\left(1-b_{j j^{\prime}}\right), \quad \forall j, j^{\prime} \in J, j<j^{\prime}, \\
& \left(s_{j^{\prime}}+h_{j^{\prime}}\right)-\left(s_{j}+h_{j}\right) \leq W+\operatorname{big} M\left(1-b_{j j^{\prime}}\right), \quad \forall j, j^{\prime} \in J, j<j^{\prime}, \\
& c_{j}=t_{i 4}+\sum_{j^{\prime} \in J_{i}, j^{\prime} \leq j} c c_{i j^{\prime}}, \quad \forall j \in J, \\
& s_{j} \geq 0, \quad \forall j \in J,
\end{aligned}
$$

where objective (13) minimizes the makespan of HR process. Constraint (14) ensures that charging time of slab $j$ is after its ready time. Constraints (15) and (16) ensure that the interval time between two continuously rolled slabs. Constraint (17) defines the variable $c_{j}$.

It is worth noting that in the practical SCC-HR process, there are four linkage modes between SCC and HR, which are continuous casting-cold charge rolling (CC-CCR) mode, continuous casting-hot charge rolling (CC-HCR) mode, continuous casting-direct hot charge rolling (CCDHCR) mode, and continuous casting-hot direct rolling (CC-HDR) mode [40]. In CC-CCR mode, the slabs usually stay in the slab yard for a few days before they are rolled. Hence, the CC-CCR slabs are not part of the integrated schedule. The CC-HDR mode requires special production line configuration, and is rarely used in steel plants. Therefore, CC-CCR and CC-HDR are not considered in our model.

For CC-HCR and CC-DHCR linkage modes, the planner will mark the corresponding slabs as CC-DHCR mode in advance according to the production process programmes. Only when the CC-DHCR mode cannot be implemented in the actual production process, the CC-DHCR slabs will be put into the heat preservation pit to slow down the temperature drop, and when the production rhythm is met, it will be charged into the heating furnace and become CCHCR slabs. Therefore, our model is suitable for CC-HCR and CC-DHCR linkage modes. 
TABLE 1: Indices, sets, parameters and decision variables.

\begin{tabular}{|c|c|}
\hline Symbol & Description \\
\hline I & Index set of heats, $I=\{1,2, \ldots,|I|\}$ \\
\hline$i, i^{\prime}$ & Index of heat, $i, i^{\prime} I$ \\
\hline$J$ & Index set of slabs, $J=\{1,2, \ldots,|J|\}$ \\
\hline$j, j^{\prime}$ & Index of slab, $j, j^{\prime} \in J$; the index is sorted according to times when slabs are cut in chronological order \\
\hline$J_{i}$ & Sub index set of slabs contained in heat $i, J_{i} \subseteq J$ \\
\hline$c c_{i j}$ & Casting duration of slab $j$ of heat $i, i \in I, j \in J_{i}$ \\
\hline$s$ & $\begin{array}{c}\text { Index of processes, } s \in\{1,2,3,4\} \text {, process } 1 \text { is steelmaking, process } 2 \text { is LF refining, process } 3 \text { is RH refining, and process } 4 \text { is } \\
\text { continuous casting }\end{array}$ \\
\hline$M^{s}$ & Index set of machines in process $s, M^{s}=\left\{1,2, \ldots,\left|M^{s}\right|\right\}, s \in\{1,2,3,4\}$ \\
\hline$m, m^{\prime}$ & Index of machine, $m, m^{\prime} \in M^{s}, s \in\{1,2,3,4\}$ \\
\hline$p_{\text {im }}$ & Processing time of heat $i$ on machine \\
\hline$\left(T_{m, i^{\prime}} T_{m, m^{\prime}}\right)$ & $\begin{array}{l}\text { Heat casting sequence relationship. } a_{i i^{\prime}}=1 \text { when the heat } i^{\prime} \text { is casted immediately after heat } i, a_{i i^{\prime}}=0 \text { otherwise } \\
\text { Minimum/maximum delay from machine } m \text { to machine } m^{\prime}, m \in M^{s}, m^{\prime} \in M^{s+1}, s=1,2,3\end{array}$ \\
\hline & Processing time of slab $j, j \in J$ in HR process \\
\hline$(Q / W)$ & Minimum $/ \mathrm{m}$ \\
\hline TS & Shortest delivery time from the positio \\
\hline$h_{j}$ & Minimum residence time of slab $j, j \in J$ in reheating furnace \\
\hline$b_{j j^{\prime}}^{\prime}$ & Slab rolling sequence relationship; $b_{i i^{\prime}}=1$ when Slab $j^{\prime}$ is rolled immediately after slab $j, b_{i i^{\prime}}=0$ otherwise \\
\hline$x_{i m}$ & $x_{i m}=1$, if heat $i$ is assigned to machine $m$, and $x_{i m}=0$, otherwise, $i \in I, m \in M^{s}, s=1,2,3,4$ \\
\hline$y_{i i^{\prime}}$ & $\begin{array}{l}y_{i i^{\prime}}=1 \text { if Heat } i \text { and heat } i^{\prime} \text { are assigned on the same machine and heat } i \text { is processed before heat } i^{\prime} \text {, and } y_{i i^{\prime}}=0 \text {, otherwise, } \\
\qquad i, i^{\prime} \in I\end{array}$ \\
\hline$t_{i s}$ & Start time of heat $i$ in process $s, i \in I, s=1,2,3,4$ \\
\hline$c_{j}$ & Time when slab $j$ is cut, $j \in J$ \\
\hline$s_{j}$ & Time when slab $j, j \in J$ is charged into reheating furnace \\
\hline$C_{1}$ & The latest finish time of all heats in the continuous casting process \\
\hline$C_{2}$ & The latest finish time of all slabs in the HR process \\
\hline
\end{tabular}

2.2.3. Integrated Mathematical Model. Considering the goal of integrated scheduling, this paper integrates the two models to establish an integrated one of SCC-HR processes and design a two-stage solving algorithm that combines genetic algorithm and mathematical programming. The integrated scheduling model is as follows.

$$
\begin{array}{ll}
\min & Z=\omega_{1} C_{1}+\omega_{2} C_{2}+\omega_{3} \sum_{j \in J}\left(s_{j}-c_{j}\right) \\
\text { s.t. } & (1) \sim(18),
\end{array}
$$

where $\omega_{1}, \omega_{2}$, and $\omega_{3}$ are weight coefficients of items $C_{1}, C_{2}$, and $\sum_{j \in J}\left(s_{j}-c_{j}\right)$ in the objective function, satisfying $\omega_{1}+\omega_{2}+\omega_{3}=1, \omega_{1}, \omega_{2}, \omega_{3}>0$. Objective (19) minimizes the weighted total time of makespan $C_{1}, C_{2}$ and waiting time of slabs in slab yard.

It is worth noting that, in fact, the sum of the weight coefficients does not necessarily equal to 1 . Taking any three numbers greater than 0 that represent the relative weights of three objective items can also realize the optimization preference. After normalizing the weight coefficients, the sum of their weights still equals 1 , so the sum of the given weights in this paper equals to 1 . In addition to the weighted sum method, there are other methods to deal with multiobjective optimization functions, such as Pareto solution set. The weighted sum method is chosen in this paper because it is simple, effective, and easy to be accepted by actual planner.

The integrated model contains two subproblems. One is an assignment problem in SCC (AG-SCC) which is to determine the processing device and sequence of each heat in each process. The other is a time optimization problem, and we call it scheduling problem in the plant-wide processes (SP-PWP). That is, under conditions that processing device and sequence of each heat in each stage have been determined, the start time of each heat in each stage and the charging time of each slab are optimized to achieve the overall optimization goal of formula (19).

After solving AG-SCC, the decision variables $x_{i m}, y_{i i^{\prime}}$ are determined, and are denoted as $\bar{x}_{i m}, \bar{y}_{i i^{\prime}}$. The SP-PWP model is a linear programming model as follows:

$$
\begin{array}{ll}
\min & (19) \\
& t_{i(s+1)}-t_{i s}-p_{i m} \geq T_{m, m^{\prime}}^{\min }, \quad \forall \bar{x}_{i m} \cdot \bar{x}_{i m^{\prime}}=1, i \in I, m \in M^{s}, m^{\prime} \in M^{s+1}, s=1,2,3 \\
& t_{i(s+1)}-t_{i s}-p_{i m} \leq T_{m, m^{\prime}}^{\max }, \quad \forall \bar{x}_{i m} \cdot \bar{x}_{i m^{\prime}}=1, i \in I, m \in M^{s}, m^{\prime} \in M^{s+1}, s=1,2,3 \\
\text { s.t. } & t_{i^{\prime} s} \geq t_{i s}+p_{i m}, \quad \forall \bar{x}_{i m}=1, \bar{y}_{i i^{\prime}}=1, i, i^{\prime} \in I, m \in M^{s}, s=1,2,3 \\
& t_{i^{\prime} 4}=t_{i 4}+p_{i m}, \quad \forall \bar{x}_{i m}=1, a_{i i^{\prime}}=1, i, i^{\prime} \in I, m \in M^{4} \\
& (1),(11),(13) \sim(18) .
\end{array}
$$




\section{Optimization Approaches}

3.1. The Two-Stage Heuristics. Considering that the integrated model is hard to solve, this paper first proposes a twostage heuristic (TSHeu). In the first stage, a priority rulebased heuristic procedure is used to solve the AG-SCC model, and the processing device and sequence of heats in each device are determined. In the second stage, a linear programming solver is used to solve the SP-PWP model, and a near-optimal solution of the integrated model is obtained.

In solving the AG-SCC, the casting tasks and executing sequence in each continuous caster have been determined. Therefore, we can calculate the starting time $s_{i m}, i \in I, m \in M^{4}$ of each heat in the casting process. In the TSHeu, the starting casting time $s_{i m}$ of each heat in the continuous caster is sorted in ascending order, and the sorted list is $P=\left(\pi_{1}, \pi_{2}, \ldots, \pi_{|I|}\right), \pi_{i} \in I$. Then, the (Algorithm 1) TSHeu is as follows.

3.2. The TSHeu-Based GA. Since the quality of solutions to the integrated model is determined by the quality of the first stage of solving the AG-SCC. This paper designs a TSHeu based genetic Algorithm 2 (TSHeu-GA) to solve the integrated problem.

The algorithm uses a mixture of roulette and optimal retention strategies when generating a new generation. The advantage of the optimal retention strategy over the traditional probability selection approach is the ability to prototype replicate the best individuals of previous generations into a new generation, thus ensuring that the best individuals produced in previous iterations are not destroyed.

3.3. Individual Coding and Fitness Calculation. As can be seen from the TSHeu that given a scheduling list $P=\left(\pi_{1}, \pi_{2}, \ldots, \pi_{|I|}\right)$, the TSHeu $(P)$ can get a solution. Therefore, the coding of the genetic algorithm takes the form of a scheduling list. The decoding process uses the heuristic algorithm TSHeu $(P)$.

Let $Z_{i}$ be the objective value of individual $I_{i}$ in population $\operatorname{Pop}(k), Z^{\max }=\max \left\{Z_{i} \mid I_{i} \in \operatorname{Pop}(k)\right\}, \quad Z^{\min }=\min \left\{Z_{i} \mid I_{i} \in\right.$ $\operatorname{Pop}(k)\}$. The fitness value $f\left(I_{i}\right)$ of individual $I_{i}$ is defined as follows:

$$
f\left(I_{i}\right)=\frac{Z^{\mathrm{max}}-Z_{i}}{Z^{\mathrm{max}}-Z^{\min }+0.01} .
$$

3.4. Initial Population Generation. Since continuous caster and casting sequence in stage 4 are determined and the earliest available time of continuous casters is 0 , then the starting time $t_{i 4}$ of heat $i, i \in I$ in stage 4 can be calculated. So, the initial population is generated as follows (Algorithm 3).

\subsection{Genetic Operators}

3.5.1. Crossover Operator. Let the two individuals involved in the crossover operation be mother $M=\left(\pi_{1}^{M}, \pi_{2}^{M}, \ldots\right.$, $\left.\pi_{|I|}^{M}\right)$ and father $F=\left(\pi_{1}^{F}, \pi_{2}^{F}, \ldots, \pi_{|I|}^{F}\right)$. Generate a random integer $p, 1<p<|I|$, two descendants of $M$ and $F$ by crossover operation at point $p$ are daughter $D=$ $\left(\pi_{1}^{D}, \pi_{2}^{D}, \ldots, \pi_{|I|}^{D}\right)$ and son $S=\left(\pi_{1}^{S}, \pi_{2}^{S}, \ldots, \pi_{|I|}^{S}\right)$. In the scheduling list of $D$, the first $p$ indexes of heats inherit from $M$, i.e.,

$$
\pi_{i}^{D}:=\pi_{i}^{M}, \quad i=1,2, \ldots, p .
$$

The indexes of heats in positions $i=p+1, \ldots,|I|$ inherit from $F$ and retain the relative positions of heats in $F$, i.e.,

$$
\begin{aligned}
\pi_{i}^{D} & :=\pi_{k}^{F}, \quad i=p+1, \ldots,|I|, \\
k & =\min \left\{k\left|\pi_{k}^{F} \notin\left\{\pi_{1}^{D}, \ldots, \pi_{i-1}^{D}\right\}, \quad k=1,2, \ldots,\right| I \mid\right\} .
\end{aligned}
$$

The scheduling list of $S$ is similar to that of $D$, i.e.,

$$
\begin{aligned}
\pi_{i}^{S} & :=\pi_{i}^{F}, \quad i=1,2, \ldots, p, \\
\pi_{i}^{S} & :=\pi_{k}^{M}, \quad i=p+1, \ldots,|I|, \\
k & =\min \left\{k\left|\pi_{k}^{M} \notin\left\{\pi_{1}^{S}, \ldots, \pi_{i-1}^{S}\right\}, \quad k=1,2, \ldots,\right| I \mid\right\} .
\end{aligned}
$$

3.5.2. Mutation Operator. Given an individual $I$, the mutation operator modifies the related heat sequence as follows. For all positions $i=1,2, \ldots,|I|-1$, heat $\pi_{i}$ and $\pi_{i+1}$ are exchanged with a probability of $p_{m}$. In our algorithms, we set $p_{m}=0.05$.

\section{Computational Results}

4.1. Testing Instances. In order to test the correctness of the model and the performance of the algorithms, we generate four problem instances by simulating the practical SCC-HR processes of a large iron \& steel plant in China. There are two BOFs, two LFs, two RHs, and two CCs in the plant. These devices constitute three process routes, which are BOF-LFCC, BOF-RH-CC, and BOF-LF-RH-CC, respectively. According to the specification of steel grade, slab sizes and product mechanical property requirement, the processing time of each heat in the devices of SCC process and the processing time of each slab on HR process is different. Among them, the processing time of each heat in BOF, LF, $\mathrm{RH}$, and $\mathrm{CC}$ is between 30 mins and 35 mins. The processing time of each slab on the hot rolling mill is about 2 mins. The interval of the transfer time between devices is shown in Table 2 .

The four problem instances include $\{10,15,20,25\}$ heats and $\{40,60,80,100\}$ slabs. The minimum interval for hot rolling is $Q=1 \mathrm{~min}$, the maximum interval is $W=10 \mathrm{mins}$, and the shortest delivery time from the position where slab is cut to reheating furnace is $T S=5$ mins.

4.2. Computational Results. The algorithm parameters are set as follows: (PopSize, Gen) $=\{(30,50),(40,38),(50,30)\}$, elitism $=5$. In order to analyze the influence of weight coefficient on optimization direction, take $\left(\omega_{1}, \omega_{2}, \omega_{3}\right)=$ $\{(0.12,0.33,0.55),(0.33,0.55,0.12),(0.55,0.12,0.33)\}$ We 
STAGE 1:

STEP 1.1: enter the list $P=\left(\pi_{1}, \pi_{2}, \ldots, \pi_{|I|}\right), \pi_{i} \in I$.

STEP 1.2: set the current time be 0 , schedule $\mathrm{RH}$ process $(s=3)$ according to $P$, if apply.

For $i=1$ to $|I|$

Select the earliest free RH device $m \in M^{3}$ to process heat $\pi_{i}$. If there are multiple RH devices idle, randomly select one.

Calculate the starting time $t_{\pi_{i} s}$ in the RH.

Resort list $P$ according to $t_{\pi_{i} s}$ in ascending order to get a new $P=\left(\pi_{1}, \pi_{2}, \ldots, \pi_{|I|}\right)$.

STEP 1.3: set the current time be 0 , schedule LF process $(s=2)$ according to $P$, if apply.

For $i=1$ to $|I|$

Select the earliest idle LF device $m \in M^{2}$ to process heat $\pi_{i}$. If there are multiple LF devices idle, choose one randomly.

Calculate the starting time $t_{\pi_{i} s}$ in the LF.

Resort list $P$ according to $t_{\pi_{i} s}$ in ascending order to get a new $P=\left(\pi_{1}, \pi_{2}, \ldots, \pi_{|I|}\right)$.

STEP 1.4: set the current time be 0 , schedule BOF process $(s=1)$ according to $P$.

For $i=1$ to $|I|$

Select the earliest free BOF device $m \in M^{1}$ to process heat $\pi_{i}$. If there are multiple BOF devices idle, randomly select one.

STAGE 2:

STEP 2.1: calculate $\bar{x}_{i m}$ and $\bar{y}_{i i^{\prime}}, \forall i, i^{\prime} \in I, m \in M^{s}, s=1,2,3$ according to the schedule.

STEP 2.2: apply linear programming solver to solve the SP-PWP model.

STEP 2.3: output the solution: $\left(\bar{x}_{i m}, \bar{y}_{i i^{\prime}}, s_{i m}, s_{j}\right)$ and the objective function value $Z$.

Algorithm 1: TSHeu $(P)$.

STEP 1: parameter initialization: population size PopSize, maximum number Gen of iterative generations, the number elitism of best individuals retained to the next generation, Current population $\operatorname{Pop}(1) \longleftarrow \phi$, Pop $(1) \longleftarrow \phi$.

STEP 2: generate an initial population Pop(1)

For $i=1$ to PopSize

Randomly generate a new individual $I$ and calculate its objective value (19).

$\operatorname{Pop}(1):=\operatorname{Pop}(1) \cup\{I\}$.

STEP 3: main loop of evolutionary process

For $k=1$ to Gen

For $i=1$ to PopSize/2

Select two individuals $I_{1}$ and $I_{2}$ from $\operatorname{Pop}(k)$ by roulette.

Apply crossover operator to $I_{1}$ and $I_{2}$ to get $I_{1}^{\prime}$ and $I_{2}^{\prime}$.

Apply mutation operator to $I_{1}^{\prime}$ and $I_{2}^{\prime}$ to get $I_{1}^{\prime \prime}$ and $I_{2}^{\prime \prime}$.

$\operatorname{Pop}^{\prime}(k)=\operatorname{Pop}^{\prime}(k) \cup\left\{I_{1}^{\prime \prime}, I_{2}^{\prime \prime}\right\}$.

Select the best elitism individuals from $\operatorname{Pop}(k) \cup \operatorname{Pop}^{\prime}(k)$ and select (PopSize - elitism) individuals from

$\operatorname{Pop}(k) \cup \operatorname{Pop} \prime(k)$ using roulette to construct a new generation $\operatorname{Pop}(k+1)$.

STEP 4: output the best solution.

Algorithm 2: TSH-GA.

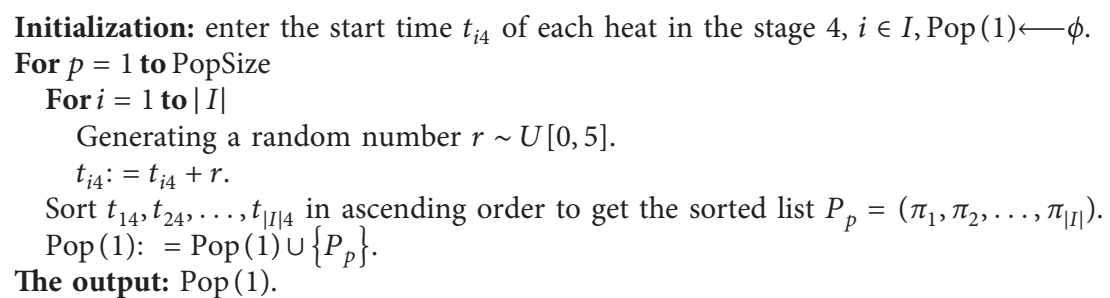

Algorithm 3: Initialization.

solved each instance 10 times and averaged the results for each instance.

In a preliminary experiment, we set $\left(\omega_{1}, \omega_{2}, \omega_{3}\right)=(0.33$, $0.33,0.34)$ to test the effect of parameter PopSize and Gen on the performance of the algorithm. The average experimental results of 10 runs for each instance are shown in Table 3 . In Table 3, the column " $P \times G$ " is the value of PopSize $\times$ Gen. The columns " $C_{1}(m)$, " " $C_{2}(m)$," "sum," "Total $(m)$ " and 
TABLe 2: The transfer time between devices.

\begin{tabular}{|c|c|c|c|c|c|c|}
\hline & LF1 & LF2 & RH1 & RH2 & $\mathrm{CC} 1$ & CC2 \\
\hline BOF1 & {$[5,9]$} & {$[6,10]$} & {$[6,10]$} & {$[7,11]$} & - & - \\
\hline BOF2 & {$[4,8]$} & {$[4,8]$} & {$[6,10]$} & {$[5,9]$} & - & - \\
\hline LF1 & - & - & {$[5,9]$} & {$[6,10]$} & {$[7,11]$} & {$[6,10]$} \\
\hline LF2 & - & - & {$[5,9]$} & {$[4,8]$} & {$[6,10]$} & {$[5,9]$} \\
\hline RH1 & - & - & - & - & {$[5,9]$} & {$[6,10]$} \\
\hline RH2 & - & - & - & - & {$[6,10]$} & {$[7,11]$} \\
\hline
\end{tabular}

TAble 3: The effect of parameter PopSize $\times$ Gen on the algorithm performance, $\left(\omega_{1}, \omega_{2}, \omega_{3}\right)=(0.33,0.33,0.34)$.

\begin{tabular}{|c|c|c|c|c|c|c|}
\hline No. & $P \times G$ & $C_{1}(m)$ & $C_{2}(m)$ & Sum & $\operatorname{Total}(m)$ & $\mathrm{CPU}(s)$ \\
\hline \multirow{3}{*}{1} & $30 \times 50$ & 395.37 & 437.32 & 307.23 & 1139.92 & 324.72 \\
\hline & $40 \times 38$ & 390.28 & 435.25 & 292.58 & 1118.11 & 330.59 \\
\hline & $50 \times 30$ & 394.59 & 437.93 & 306.09 & 1138.61 & 327.67 \\
\hline \multirow{3}{*}{2} & $30 \times 50$ & 558.73 & 599.25 & 648.30 & 1806.28 & 475.60 \\
\hline & $40 \times 38$ & 557.85 & 591.30 & 628.27 & 1777.42 & 487.37 \\
\hline & $50 \times 30$ & 563.96 & 606.72 & 647.25 & 1817.93 & 481.45 \\
\hline \multirow{3}{*}{3} & $30 \times 50$ & 642.27 & 696.19 & 731.35 & 2069.81 & 653.56 \\
\hline & $40 \times 38$ & 633.45 & 686.27 & 715.27 & 2034.99 & 662.52 \\
\hline & $50 \times 30$ & 643.38 & 695.41 & 732.87 & 2071.66 & 656.25 \\
\hline \multirow{3}{*}{4} & $30 \times 50$ & 820.29 & 872.61 & 828.32 & 2521.22 & 792.26 \\
\hline & $40 \times 38$ & 815.36 & 861.45 & 816.45 & 2493.26 & 797.53 \\
\hline & $50 \times 30$ & 819.58 & 859.78 & 821.68 & 2501.04 & 795.96 \\
\hline
\end{tabular}

TABLE 4: Effect of different weight coefficient combinations on $C_{1}, C_{2}$, and $\sum_{j \in J}\left(s_{j}-c_{j}-h_{j}\right)$ indicators.

\begin{tabular}{lccccccc}
\hline No. & $\left(\omega_{1}, \omega_{2}, \omega_{3}\right)$ & $C_{1}(m)$ & $C_{2}(m)$ & Sum & TSHGA & Ran & Ratio $(\%)$ \\
\hline \multirow{3}{*}{1} & $(0.12,0.33,0.55)$ & 405.32 & 435.76 & 257.14 & 333.87 & 393.95 \\
& $(0.33,0.55,0.12)$ & 391.39 & 423.12 & 308.57 & 398.90 & 440.32 & 18.00 \\
& $(0.55,0.12,0.33)$ & 375.27 & 450.19 & 278.01 & 352.16 & 407.50 & 15.72 \\
\hline \multirow{2}{*}{2} & $(0.12,0.33,0.55)$ & 570.12 & 596.35 & 635.31 & 614.63 & 748.05 & 21.71 \\
& $(0.33,0.55,0.12)$ & 553.67 & 582.13 & 645.28 & 580.32 & 691.81 & 19.21 \\
\hline \multirow{3}{*}{3} & $(0.55,0.12,0.33)$ & 546.75 & 623.71 & 641.17 & 587.14 & 649.26 & 10.58 \\
\hline & $(0.12,0.33,0.55)$ & 655.38 & 694.27 & 720.25 & 703.89 & 870.07 & 23.61 \\
4 & $(0.33,0.55,0.12)$ & 631.37 & 673.38 & 730.89 & 666.42 & 736.37 & 10.50 \\
& $(0.55,0.12,0.33)$ & 625.19 & 705.57 & 725.18 & 667.83 & 757.55 & 13.44 \\
\hline
\end{tabular}

"CPU $(s)$ " report the values of $C_{1}, C_{2}, \sum_{j \in J}\left(s_{j}-c_{j}\right)$, $C_{1}+C_{2}+\sum_{j \in J}\left(s_{j}-c_{j}\right)$, and averaged run time, respectively.

It can be seen from Table 3 that when the weight coefficient is set to $\left(\omega_{1}, \omega_{2}, \omega_{3}\right)=(0.33,0.33,0.34)$ and $($ PopSize, Gen $)=(40,38)$, the algorithm perform best. Therefore, in the further experiment, the algorithm parameters are set as (PopSize, Gen) $=(40,38)$, and the weight coefficients of items in the objective function are analysed to observe the influence on each objective item.

In order to test the effectiveness of the algorithm TSHeuGA, we randomly generated the same number of solutions in the experiment and compared the values of the objective function under different weight coefficients combinations. When the algorithm TSHeu-GA is applied to solve an instance, the number of generated solutions per run is $40 \times 38=1520$. Therefore, in the experiment, we randomly generated 1520 solutions and calculated the value of objective function under different weight coefficients combinations and selected the best one for comparison. The generation process of the random solution is as follows. First, we use the algorithm Initialization to generate 1520 individuals. Secondly, we use the algorithm TSHeu to decode each individual, so as to obtain the values of the three items of the objective function of each individual. Thirdly, we calculate the value of objective function under different weight coefficients combinations.

The average experimental results of 10 runs of TSHeuGA and the best solution among the randomly generated 1520 solutions for each instance under different weight coefficients combinations are shown in Table 4. In Table 4, the column "TSHGA" represents the value of the objective function (19) obtained by the algorithm TSHeu-GA. The column "Ran" represents the minimum of the 1520 solutions generated from the objective function (19) under the 
same weight coefficients combinations. The column "ratio" is the improved ratio of the solution of algorithm TSHeu-GA to the randomly generated solution. The calculation formula is as follows:

$$
\text { ratio }=\frac{\text { Ran }- \text { TSHGA }}{\text { TSHGA }} \times 100 \% .
$$

It can be seen from Table 4 that (1), with the same consumption of computation resources of generating the same number of solutions, the results of the algorithm TSHeu-GA are significantly better than those of random method, indicating that the algorithm mechanism in this paper can effectively improve the quality of solutions. (2) When the weight coefficients of items in the objective function are changed, there are obvious changes in the values of items, which indicates that the algorithm can be optimized to different optimization directions by adjusting the weight coefficients of each item. This is very helpful in application.

\section{Conclusions}

In this paper, aiming at the integrated scheduling problem of SCC-HR processes, the energy saving goal is converted to minimize waiting time for slabs in slab yard, and an integrated mixed integer linear programming model is established. A two-stage heuristic TSHeu is designed for solving the difficult model. Furthermore, based on the TSHeu, a genetic algorithm is designed. The computational results shown that the models and algorithms are correct and effective, and the algorithms can be guided to optimize to different optimization directions.

In the future research, the optimization method of integrated scheduling for SCC-HR processes under the condition of stepped electricity price can be considered. It is also needed to consider the improvement of the models and algorithms in the case where the processing time of each heat is controllable within a time interval.

\section{Data Availability}

The experimental data of this study are available from the corresponding author upon request.

\section{Conflicts of Interest}

The authors declare that they have no conflicts of interest.

\section{Acknowledgments}

This work was supported in part by the Ministry of Industry and Information Technology of People's Republic of China for the key project "The construction of professional CPS test and verification bed for the application of steel rolling process" (no. TC17085JH).

\section{References}

[1] G. Feng, "Analysis of energy saving technology for steel rolling system," China Metallurgy, vol. 16, no. 11, pp. 37-40, 2006.
[2] American Iron and Steel Institute, Saving One Barrel of Oil Per Ton-A New Roadmap for Transformation of Steelmaking Process, American Iron and Steel Institute, Washington, DC, USA, 2005, https://steel.org/ /media/Files/AISI/Public\% 20Policy/saving_one_barrel_oil_per_ton.pdf.

[3] M. Biondi, S. Saliba, and I. Harjunkoski, "Production optimization and scheduling in a steel plant: hot rolling mill," in Proceedings of the 18th IFAC, pp. 11750-11754, Milano, Italy, 2011.

[4] L. Tang and G. Wang, "Decision support system for the batching problems of steelmaking and continuous-casting production," Omega, vol. 36, no. 6, pp. 976-991, 2008.

[5] P. I. Cowling, D. Ouelhadj, and S. Petrovic, "Dynamic scheduling of steel casting and milling using multi-agents," Production Planning \& Control, vol. 15, no. 2, pp. 178-188, 2004.

[6] L. Tang, J. Liu, A. Rong, and Z. Yang, "A mathematical programming model for scheduling steelmaking-continuous casting production," European Journal of Operational Research, vol. 120, no. 2, pp. 423-435, 2000.

[7] K. Mao, Q.-k. Pan, X. Pang, and T. Chai, "A novel Lagrangian relaxation approach for a hybrid flowshop scheduling problem in the steelmaking-continuous casting process," European Journal of Operational Research, vol. 236, no. 1, pp. 51-60, 2014.

[8] J. Li, X. Xiao, Q. Tang, and C. A. Floudas, "Production scheduling of a large-scale steelmaking continuous casting process via unit-specific event-based continuous-time models: short-term and medium-term scheduling," Industrial \& Engineering Chemistry Research, vol. 51, no. 21, pp. 73007319, 2012.

[9] A. Bellabdaoui and J. Teghem, "A mixed-integer linear programming model for the continuous casting planning," International Journal of Production Economics, vol. 104, no. 2, pp. 260-270, 2006.

[10] Q.-K. Pan, "An effective co-evolutionary artificial bee colony algorithm for steelmaking-continuous casting scheduling," European Journal of Operational Research, vol. 250, no. 3, pp. 702-714, 2016.

[11] P. A. Huegler and F. J. Vasko, "Metaheuristics for meltshop scheduling in the steel industry," Journal of the Operational Research Society, vol. 58, no. 6, pp. 791-796, 2007.

[12] L. Tang, J. Guan, and G. Hu, "Steelmaking and refining coordinated scheduling problem with waiting time and transportation consideration," Computers \& Industrial Engineering, vol. 58, no. 2, pp. 239-248, 2010.

[13] L. Tang and X. Wang, "An improved particle swarm optimization algorithm for the hybrid flow shop scheduling to minimize total weighted completion time in process industry," IEEE Transactions on Control Systems Technology, vol. 18, no. 6, pp. 1303-1314, 2010.

[14] S. Jiang, M. Liu, J. Hao, and W. Qian, “A bi-layer optimization approach for a hybrid flow shop scheduling problem involving controllable processing times in the steelmaking industry," Computers \& Industrial Engineering, vol. 87, pp. 518-531, 2015.

[15] M. Yadollahpour and B. ArbabShirani, "A comprehensive solution for continuous casting production planning and scheduling," The International Journal of Advanced Manufacturing Technology, vol. 82, no. 1-4, pp. 211-226, 2016.

[16] A. Atighehchian, M. Bijari, and H. Tarkesh, "A novel hybrid algorithm for scheduling steel-making continuous casting production," Computers \& Operations Research, vol. 36, no. 8, pp. 2450-2461, 2009. 
[17] Y. Ye, J. Li, Z. Li, Q. Tang, X. Xiao, and C. A. Floudas, "Robust optimization and stochastic programming approaches for medium-term production scheduling of a large-scale steelmaking continuous casting process under demand uncertainty," Computers \& Chemical Engineering, vol. 66, pp. 165-185, 2014.

[18] J. Hao, M. Liu, S. Jiang, and C. Wu, "A soft-decision based two-layered scheduling approach for uncertain steelmakingcontinuous casting process," European Journal of Operational Research, vol. 244, no. 3, pp. 966-979, 2015.

[19] Y.-y. Tan, Y.-1. Huang, and S.-x. Liu, “Two-stage mathematical programming approach for steelmaking process scheduling under variable electricity price," Journal of Iron and Steel Research International, vol. 20, no. 7, pp. 1-8, 2013.

[20] Y. Tan and S. Liu, "Models and optimisation approaches for scheduling steelmaking-refining-continuous casting production under variable electricity price," International Journal of Production Research, vol. 52, no. 4, pp. 1032-1049, 2014.

[21] H. Hadera, I. Harjunkoski, G. Sand, I. E. Grossmann, and S. Engell, "Optimization of steel production scheduling with complex time-sensitive electricity cost," Computers \& Chemical Engineering, vol. 76, pp. 117-136, 2015.

[22] D. Gajic, H. Hadera, L. Onofri, I. Harjunkoski, and S. Di Gennaro, "Implementation of an integrated production and electricity optimization system in melt shop," Journal of Cleaner Production, vol. 155, pp. 39-46, 2017.

[23] J. Long, Z. Sun, P. M. Pardalos, Y. Hong, S. Zhang, and C. Li, "A hybrid multi-objective genetic local search algorithm for the prize-collecting vehicle routing problem," Information Sciences, vol. 478, pp. 40-61, 2019.

[24] Q.-K. Pan, Q.-d. Chen, T. Meng, B. Wang, and L. Gao, “A mathematical model and two-stage heuristic for hot rolling scheduling in compact strip production," Applied Mathematical Modelling, vol. 48, pp. 516-533, 2017.

[25] C. Pan and G. K. Yang, "A method of solving a large-scale rolling batch scheduling problem in steel production using a variant of column generation," Computers \& Industrial Engineering, vol. 56, no. 1, pp. 165-178, 2009.

[26] T. Zhang, W. Art Chaovalitwongse, Y.-J. Zhang, and P. M. Pardalos, "The hot-rolling batch scheduling method based on the prize collecting vehicle routing problem," Journal of Industrial \& Management Optimization, vol. 5, no. 4, pp. 749-765, 2009.

[27] L. Lopez, M. W. Carter, and M. Gendreau, "The hot strip mill production scheduling problem: a tabu search approach," European Journal of Operational Research, vol. 106, no. 2-3, pp. 317-335, 1998.

[28] L. Tang and X. Wang, "A scatter search algorithm for a multistage production scheduling problem with blocking and semi-continuous batching machine," IEEE Transactions on Control Systems Technology, vol. 19, no. 5, pp. 976-989, 2011.

[29] Y.-W. Chen, Y.-Z. Lu, M. Ge, G.-K. Yang, and C.-C. Pan, "Development of hybrid evolutionary algorithms for production scheduling of hot strip mill," Computers \& Operations Research, vol. 39, no. 2, pp. 339-349, 2012.

[30] J. Zhao, W. Wang, Q. Liu, Z. Wang, and P. Shi, "A two-stage scheduling method for hot rolling and its application," Control Engineering Practice, vol. 17, no. 6, pp. 629-641, 2009.

[31] L. Tang and X. Wang, "A two-phase heuristic for the production scheduling of heavy plates in steel industry," IEEE Transactions on Control Systems Technology, vol. 18, no. 1, pp. 104-117, 2010.

[32] S. J. Jia, J. Yi, G. K. Yang, B. Du, and J. Zhu, "A multi-objective optimisation algorithm for the hot rolling batch scheduling problem," International Journal of Production Research, vol. 51, no. 3, pp. 667-681, 2013.

[33] M. Yadollahpour, M. Bijari, S. Kavosh, and M. Mahnam, "Guided local search algorithm for hot strip mill scheduling problem with considering hot charge rolling," International Journal of Advanced Manufacturing Technology, vol. 45, no. 11-12, pp. 1215-1231, 2009.

[34] C. Xu, G. Sand, I. Harjunkoski, and S. Engell, "A new heuristic for plant-wide schedule coordination problems: the intersection coordination heuristic," Computers \& Chemical Engineering, vol. 42, pp. 152-167, 2012.

[35] Y. Tan, S. Liu, and Y. Huang, "A hybrid approach for the integrated scheduling of steel plants," ISIJ International, vol. 53, no. 5, pp. 848-853, 2013.

[36] J. Lin, M. Liu, J. Hao, and S. Jiang, "A multi-objective optimization approach for integrated production planning under interval uncertainties in the steel industry," Computers \& Operations Research, vol. 72, pp. 189-203, 2016.

[37] J. Lin, M. Liu, J. Hao, and P. Gu, "Many-objective harmony search for integrated order planning in steelmaking-continuous casting-hot rolling production of multi-plants," International Journal of Production Research, vol. 55, no. 14, pp. 4003-4020, 2017.

[38] I. Mattik, P. Amorim, and H.-O. Günther, "Hierarchical scheduling of continuous casters and hot strip mills in the steel industry: a block planning application," International Journal of Production Research, vol. 52, no. 9, pp. 2576-2591, 2014.

[39] V. Jain and I. E. Grossmann, "Algorithms for hybrid MILP/ CP models for a class of optimization problems," INFORMS Journal on Computing, vol. 13, no. 4, pp. 258-276, 2001.

[40] L. Tang, J. Liu, A. Rong, and Z. Yang, "A review of planning and scheduling systems and methods for integrated steel production," European Journal of Operational Research, vol. 133, no. 1, pp. 1-20, 2001. 